\section{ATG-101, A NOVEL PD-L1/4-1BB BISPECIFIC ANTIBODY, AUGMENTS ANTI-TUMOR IMMUNITY THROUGH IMMUNE CHECKPOINT INHIBITION AND PDL1-DIRECTED 4-1BB ACTIVATION}

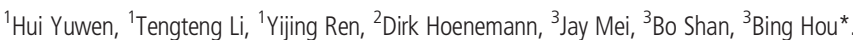
${ }^{1}$ Shanghai Antengene Corporation Limited., Shanghai, China; ${ }^{2}$ Antengene Pty Ltd, Melbourne, Australia; ${ }^{3}$ Antengene Corporation Co., Ltd., Shao xing, China

Background Programmed death-ligand 1 (PD-L1) and programmed cell death protein $1(\mathrm{PD}-1)$ blockade therapy has revolutionized the treatment landscape of malignancies. However, only a minority of patients are anticipated to experience a deep and durable response. In addition, successful therapeutic agonism of $4-1 \mathrm{BB}$, a promising co-stimulatory immunologic target, has been limited by major safety concerns of hepatotoxicity or suboptimal agonistic potency. ATG-101, a novel PD-L1/4-1BB bispecific antibody, was designed to activate 4$1 \mathrm{BB}$ positive $\mathrm{T}$ cells in a PDL1-crosslinking dependent manner and to effectively treat tumors without on-target-off-tumor liver toxicity (figure 1).

Methods ATG-101 was developed by introducing lower affinity $4-1 \mathrm{BB}$ scFv into a human IgG1 PD-L1 monoclonal antibody. The N297A mutation on $\mathrm{CH} 2$ abolishes the binding capacity to most FcyRs but retains the binding to FcyRn. A series of in vitro and in vivo studies were performed to evaluate the potency, safety and specific mechanism of action.

Results ATG-101 simultaneous binds to 4-1BB and PD-L1 with higher affinity to PD-L1, and potently activates 4-1BB positive $\mathrm{T}$ cells when crosslinked by PD-L1 positive cells. Upon crosslinking, ATG-101 also activates PD1+TIM3+ exhausted $\mathrm{T}$ cells in vitro, suggesting a potential in reversing T-cell dysfunction and exhaustion (figure 1). ATG-101 shows potent anti-tumor activities in various animal models, including h4-1BB humanized mice bearing MC38 colon cancer, PD(L)1 blockade insensitive B16F10 melanoma and EL4 lymphoma, with no body weight loss observed. To evaluate ATG-101 efficacy in tumors progressing after anti- $\mathrm{PD}(\mathrm{L}) 1$ treatment, mice bearing MC38 tumors were treated with anti-PDL1 initially to achieve tumor growth inhibition, and half of the mice switched to ATG-101 upon disease progression, the other mice continuing with anti-PD-L1 treatment. ATG-101 induced potent tumor growth inhibition and tumor regression in antiPDL1-resistant tumors and prolonged survival. Flow cytometry and multiplex IHC staining of tumor samples from mice treated with ATG-101 or control suggest that ATG-101 increases the infiltration, proliferation and activation of CD8+ $\mathrm{T}$ cells (figure 2), the infiltration of natural killer $\mathrm{T}$ cells and the CD8+/Treg ratio in TILs. In a 4-week GLP toxicity study in cynomolgus monkey, up to $100 \mathrm{mg} / \mathrm{kg}$ repeated doses of ATG-101 were well tolerated with no hepatotoxicity observed. Conclusions ATG-101 demonstrated significant anti-tumor activity in various tumor models including those progressing on anti $\mathrm{PD}(\mathrm{L}) 1$ treatment. Good safety and $\mathrm{PK} / \mathrm{PD}$ properties has been demonstrated in preclinical in vivo models. A phase I, multicenter, dose-escalating clinical trial evaluating ATG-101 in patients with solid tumors and hematologic malignancies is ongoing.

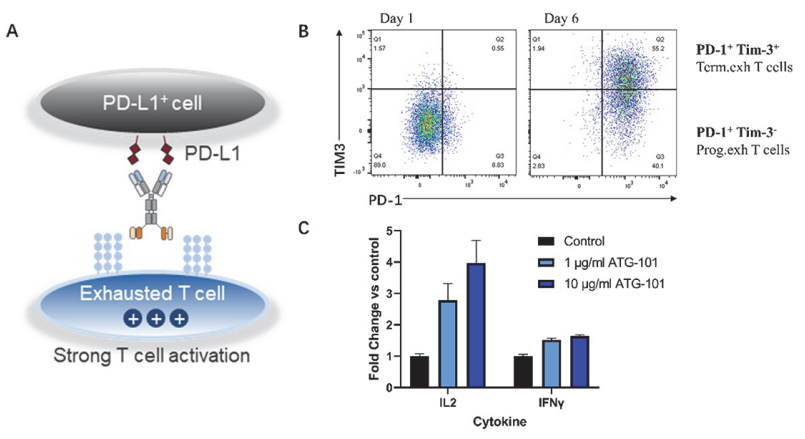

Abstract 893 Figure 1 ATG-101 conditionally activates exhausted T cells. (A) Mechanism of action of ATG-101 (B) Exhausted T cells were induced by $C D 3+T$ cells cultured with anti-CD3/CD28 beads for 6 days. The percentage of terminally exhausted T cells (PD-1+Tim-3+) and progenitor exhausted T cells (PD-1+Tim-3-) were increased on Day6 (C) With the presence of PD-L1 positive cells, ATG-101 induced the IL2 and INF- $\gamma$ secretion by exhausted T cells.
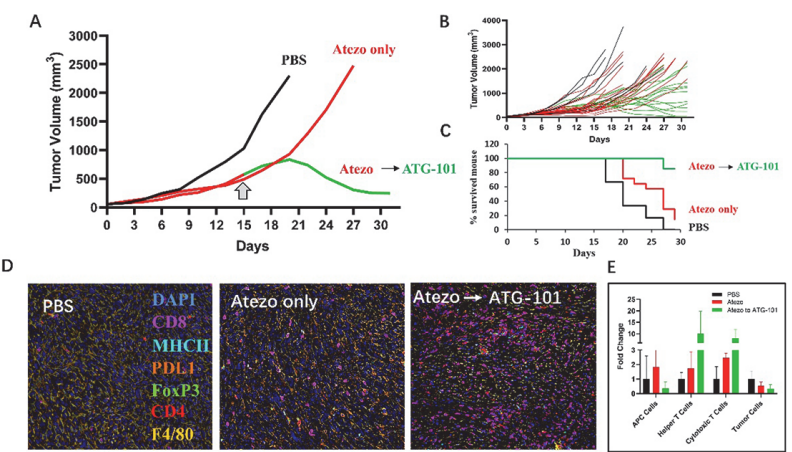

Abstract 893 Figure 2 Potent in vivo efficacy of ATG-101. (A) Representative MC38 tumor growth curve for individual mice treated with PBS (black), 10mpk atezolizumab (Atezo) only (red) or mice initially treated with $10 \mathrm{mpk}$ atezolizumab and switched to $13 \mathrm{mpk}$ ATG101 upon disease progression (red-green); the arrow indicates the day switching Atezo to ATG-101; (B) MC38 tumor growth curve for all individual mice treated with PBS (black, $n=6$ ), atezolizumab only (red, $\mathrm{n}=14$ ), and atezolizumab initially before switching to ATG-101 upon disease progression (red-green, $n=14$ ) ; (C) Survival data of mouse shown in (B); (D) Representative images for multiplex IHC staining of tumor samples collected from mouse from (B). (E) Quantitative analysis of TILs shown in (D). Compared with PBS group or atezo-only group, ATG-101 significantly increased the infiltration of T cells in the tumor microenvironment. MHCIl: APC Cells, CD4+ CD8-: Helper T Cells, CD4CD8+: Cytotoxic T Cells, CD4- CD8- F4/80- PDL1+: Tumor Cells. 
Ethics Approval The protocol and any amendment(s) or procedures involving the care and use of animals in this study were reviewed and approved by the Institutional Animal Care and Use Committee (IACUC) of CrownBio or Innostar prior to execution with an AUP number or IACUC approval number for each animal study. During the study, the care and use of animals were conducted in accordance with the regulations of the Association for Assessment and Accreditation of Laboratory Animal Care (AAALAC).All studies were conducted following an approved IACUC protocol. AUP NO.:2004-121465, 2004-12-1000; IACUC approval number: IACUC-2021M-003

http://dx.doi.org/10.1136/jitc-2021-SITC2021.893 